\title{
SISTEMAS DE INFORMACIÓN GEOGRÁFICA Y SU APLICACIÓN EN LAS CIENCIAS SOCIALES: UNA REVISIÓN BIBLIOGRÁFICA
}

\author{
GEOGRAPHICAL INFORMATION SYSTEMS AND THEIR APPLICA- \\ TION IN SOCIAL SCIENCES: A BIBLIOGRAPHICAL REVIEW
}

\section{RESUMEN}

Este artículo presenta una revisión bibliográfica sobre la evolución de los Sistemas de Información Geográfica (SIG) y su integración en las ciencias sociales, esto es importante debido a que la interrelación de estas áreas, aporta al conocimiento de los pueblos. En este sentido, el objetivo planteado fue contribuir al conocimiento académico universitario, a través de la recopilación, clasificación, análisis y síntesis de obras científicas acordes a la temática tratada. Para este fin se utilizaron los métodos histórico, sintético, dialéctico, y analítico, con un tipo de investigación descriptiva y documental, obteniendo como resultado que los SIG son de gran utilidad en diferentes campos de las ciencias sociales, que van desde la arqueología hasta la sociología, pasando por temas tan concretos como la economía y la criminología.

Palabras clave: Sistemas de Información Geográfica, Ciencias Sociales, Tecnologías de Información y Comunicación

\section{ABSTRACT}

This article presents a bibliographical review on the evolution of Geographic Information Systems (GIS) and their integration in the social sciences, this is important because the interrelation of these branches contributes to the knowledge of the peoples. In this sense, the objective was to contribute to university academic knowledge, through the collection, classification, analysis and synthesis of scientific works according to the subject matter. To this end, historical, synthetic, dialectical, and analytical methods were used, with a type of descriptive and documentary research, obtaining as a result that GIS are very useful in different fields of social sciences, which encompasses from archeology to sociology, including other such as economics and criminology.

Keywords: Geographic Information Systems, Social Sciences, Information and Communication Technologies
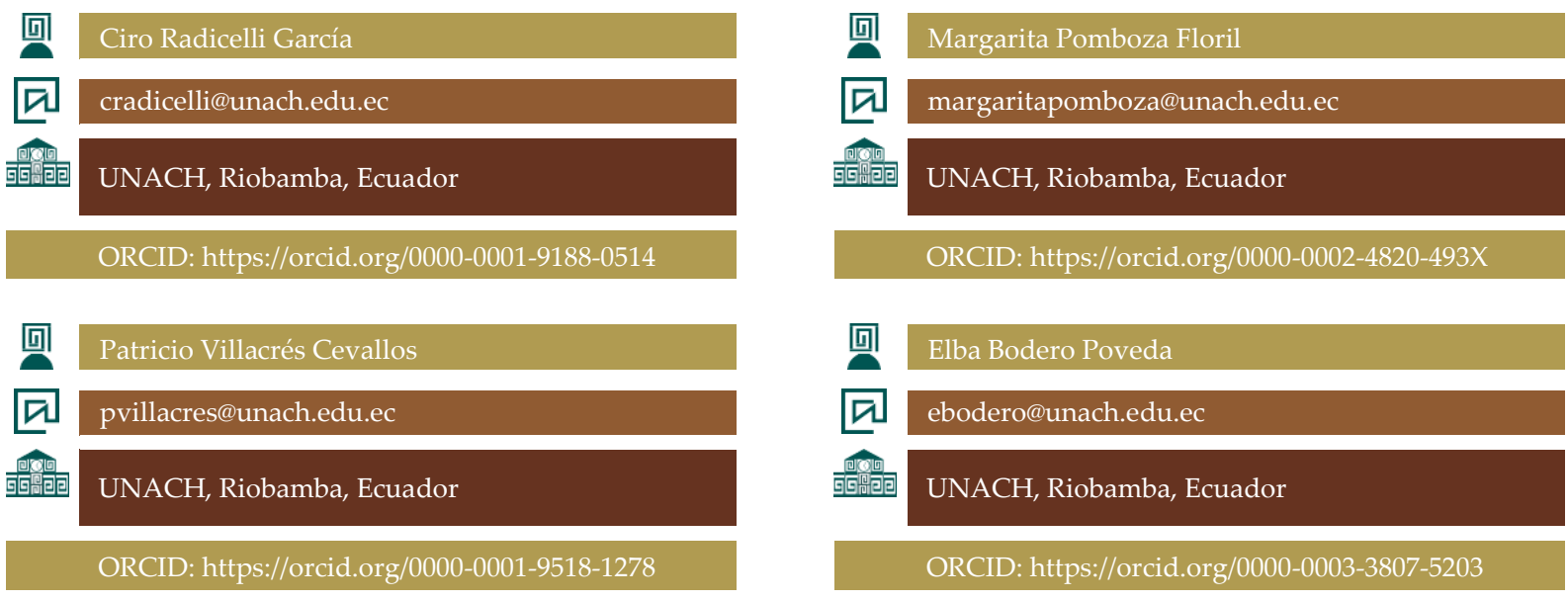


\section{INTRODUCCIÓN}

Desde tiempos inmemorables, el ser humano ha sentido la necesidad de descubrir nuevos horizontes y plasmó dichos descubrimientos de diferentes maneras, así el hombre de Cromagnon (homo sapiens europeo), realizaba pinturas rupestres sobre las rutas de los animales que cazaba 13.000 años antes de Cristo (a.C). Mucho tiempo después, en el siglo XVIII, un devastador brote de cólera suscitado en Londres, hizo que el médico John Snow ayudado de la cartografía, elabore un mapa de un área de la ciudad londinense (Cerda \&Valdivia 2007), mediante el cual detectó el problema y pudo hallar la solución. En el siglo XIX específicamente en el año de 1962, Roger Tomlinson, crea el primer SIG en Canadá (Aguirre 2013), razón por la cual se lo considera como el padre de los SIG, y es en base a este estudio que en ese mismo año aparece el Instituto de Investigación de Sistemas Ambientales - Environmental Systems Research Institute - ESRI por sus siglas en inglés, quienes actualmente desarrollan aplicaciones para SIG (ESRI 2017). En la actualidad, el avance de los SIG ha sido vertiginoso, sobre todo con el uso de internet como plataforma masiva de comunicación y manejo de datos geográficos. Durante décadas los SIG se han aplicado entre otras cosas para:

resolver problemas de gestión territorial y de recursos naturales, asuntos relacionados con el medio ambiente,

(ii) logística militar,

(iii) ciencias de la tierra (geología, geografía, etc),

(iv) navegación,

(v) arquitectura, entre otras.

"Sólo recientemente se ha empezado a considerar el uso del potencial de los SIG para otros campos y disciplinas relativamente inéditos y en particular en la investigación en Ciencias Humanas y Sociales" (Del Bosque, Fernández, Martín-Forero \& Pérez
2012:13).

En este sentido, un SIG no es más que la agrupación de datos, procedimientos, hardware, software y recursos humanos (Santovenia, Tarragó \& Cañedo 2009), que se utilizan para trabajar con datos geográficos, y que generalmente se aplican a sistemas informáticos, los cuales son utilizados entre otras cosas para la investigación en ciencias de la tierra (Alonso 2006). Así es importante mencionar que un SIG no es solamente una base de datos, aunque utiliza esta herramienta informática para almacenar la información obtenida.

Un claro ejemplo se lo puede encontrar en el estudio planteado por Maldonado (2012), quien propone la utilización de los SIG para enseñar ciencias sociales a jóvenes que cursan estudios en el nivel secundario, con lo cual se pretende motivar la investigación, aprovechando las potencialidades de los sistemas geográficos.

Bajo estos precedentes, el objetivo de este estudio es aportar al conocimiento académico universitario mediante la recopilación, clasificación, análisis y síntesis de trabajos científicos relevantes sobre la aplicación de los SIG en diversas áreas de las ciencias sociales. Es importante mencionar que esta investigación se llevó a cabo debido al creciente uso de los SIG en diferentes disciplinas académicas, donde por supuesto las relacionadas con los estudios sociales no son la excepción, además de que puede servir para aquellos profesionales que buscan nuevas áreas de trabajo para la aplicación de los SIG. Sin embargo, a pesar de lo expresado anteriormente las investigaciones sobre aspectos sociales, utilizando como herramienta de apoyo a los SIG no son muy conocidas, razón por la cual se decidió escribir el artículo en mención.

\section{METODOLOGÍA}

El artículo presentado corresponde a una revisión bibliográfica, a fin de definir la aplicación de los SIG en las ciencias sociales, 
donde se utilizaron los siguientes métodos: Histórico, debido a que en primer lugar se definió la evolución de los SIG;

1. Sintético, puesto que se identificaron los elementos de los SIG que podrían utilizarse para su aplicación en las ciencias sociales;

2. Dialéctico, debido a que se consideraron los fenómenos históricos que involucraron el desarrollo de los SIG, y

3. Analítico debido a que se distinguió y ordenó cada elemento de los SIG para aplicarlos a las ciencias sociales.

En el artículo, se detalla, previo a una revisión de la literatura publicada sobre el tema, los beneficios de la aplicación de los SIG en las ciencias sociales. Para este efecto, en primer lugar, se revisó documentación referente a sistemas de información en general y a los SIG en particular, para determinar su evolución a lo largo de tiempo. Después se revisaron las aplicaciones en las cuales los SIG han aportado.

\section{RESULTADOS Y DISCUSIÓN}

La aplicación de los SIG en las ciencias sociales tiene un importante referente en los trabajos desarrollados por el Centro de Ciencias Sociales Espacialmente Integrados - Center for Spatially Integrated Social Sciences- CSISS por sus siglas en inglés, donde a través del uso de tecnologías de la información y comunicación (TIC) relativos a técnicas geoespaciales y de georreferenciación, se han utilizado los SIG vinculados a ciencias de carácter social como las siguientes:

Arqueología, desde la gestión de recursos arqueológicos hasta la concepción de la arqueología del paisaje. En este sentido existen algunos estudios base como por ejemplo el propuesto por Figuerero e
Izeta (2013), acerca del uso de SIG en la arqueología, en donde se analiza cuan prevalente es el uso de estos sistemas en la arqueología de América del Sur, y en donde se obtuvo como resultado que existe una tendencia de mayor autonomía por parte de los arqueólogos en cuanto a la utilización de los SIG.

Así mismo se tiene el estudio propuesto por Pastor, Murrieta y García (2013) sobre la utilización de los SIG en la arqueología de habla hispana, donde justamente se analiza la situación vigente de la arqueología en Iberoamérica, obteniéndose como resultado que el desarrollo de los SIG así como los métodos y técnicas para el análisis arqueológico espacial ha sido bastante variable.

Se cuenta también con el estudio presentado por Rodríguez-Pérez (2015) en cuanto al uso de bases de datos y Sistemas de Información Geográfica para el estudio arqueológico de un tipo específico de arquitectura como la vernácula. Incluso en este campo se habla de la utilización de los SIG en el análisis arqueofaunístico, como una herramienta de apoyo para examinar el espacio y los recursos (Sartori, Fernando \& Colasurdo 2017), y es que indudablemente existe una fuerte correlación entre la arqueología y los SIG, debido a que esta última es utilizada como una herramienta de análisis e interpretación del registro arqueológico, lo que se puede evidenciar en Figuerero (2018), a través de su estudio sobre tecnología e ideas usadas para el análisis espacial de paisajes arqueológicos en la Argentina, o en el estudio propuesto por Ibarra (2013), en cuanto a las aplicaciones del sistema de información geo referenciado en el Ecuador, donde concluye que este tipo de sistemas son utilizados en investigaciones científicas arqueológicas.

Antropología, debido a la realización de estudios como el planteado por 
Carrera (2007) en el que explica comola cartografía etnográfica es utilizada como una herramienta técnica metodológica en investigaciones antropológicas, o el definido por Rivas y Perera (2008) en el cual hacen alusión al uso de la etnocartografía para el cuidado del medioambiente, o el planteado por Malo (2009), en donde analizar el uso de los SIG para la planificación adecuada del territorio, a fin de obtener un desarrollo sustentable de sus habitantes, en donde se concluye que una buena forma de alcanzar dicho desarrollo es, entre otras cosas, mediante la construcción de modelos territoriales a través de la generación de mapas.

En el caso de nuestro país, se encuentra la obra de Enríquez et al. (2011), denominada: La arqueología y la antropología en Ecuador: Escenarios, retos perspectivas, donde se analizan diversos escenarios en los cuales los SIG y otras herramientas tecnológicas pueden ser utilizadas a fin de plantear nuevos estudios en cuanto a arqueología y antropología en el país.

Por otro lado, existen estudios que relacionan a laingeniería con la antropología para establecer por ejemplo un sistema de indicadores sobre transporte público y movilidad (Hernández \& Witter 2011), donde se explica la importancia de los SIG en el análisis sobre el territorio para el diseño e implementación de servicios de transporte eficiente. Y de hecho este concepto no es nuevo, García (1976) hablaba sobre la antropología del territorio y del efecto que ejercen sobre la sociedad diversos factores como la biodiversidad, el patrimonio cultural, el conocimiento, entre otros (Ther 2012). Economía, puesto que se relaciona con investigaciones sobre el crecimiento económico, la economía ambiental, el uso de la tierra, y otros como los estudios de distribución de la población, fertilidad, planificación familiar, y demás temas relacionados con la demografía. Aquí se pueden encontrar estudios como el de Saldarriaga (2007), sobre el uso de los SIG para la conservación del medio ambiente, o el de Haro-Martínez y Taddei-Bringas (2014), quienes explican a través del uso de los SIG, que tan sustentable puede ser una economía de acuerdo con su valoración ambiental.

Se encuentra además el estudio realizado por Goerlich y Cantarino (2013), quienes hacen uso de los SIG para realizar la distribución de la población sobre un territorio determinado, con el objetivo de estudiar las relaciones entre economía $\mathrm{y}$ medio ambiente en un sistema unificado. De la misma manera se cuenta con el estudio de Fagandini y Villanueva (2012), sobre la utilización de los SIG como una herramienta que facilite la toma de decisiones territoriales en cuanto a la planificación, ordenación y gestión integral de los mismos.

EnelEcuador, existe un estudio publicado por el Programa de las Naciones Unidas para el Desarrollo (PNUD), en donde se realiza un análisis situacional de la soberanía alimentaria en el contexto de la adaptación al cambio climático, en donde se utilizan los SIG para obtener datos de producción en determinados sectores productivos, con lo que se pretende "reducir la vulnerabilidad de los cultivos más importantes para la economía ecuatoriana" (Rosero, Vásquez \& Cordero 2010).

Geografía humana, debido a la relación entre el ser humano y el mundo que lo rodea. Se enfoca además en el movimiento migratorio, cambio climático, planeamiento territorial, geopolítica, entre otros. Claros ejemplos se pueden observar en el estudio de los SIG como instrumento para el análisis de las migraciones, el ejemplo del éxodo catalán de 1936 propuesta por Burel, Rubió y Sitjár (2012), donde se analizan a través del uso de cartografía los senderos tomados por los migrantes a través de los 
Pirineos, con lo cual la representación cartográfica asistida por los SIG es clave para demostrar la incidencia del desplazamiento poblacional.

En este contexto, se encuentra también el estudio de Alonso (2004), quien habla de la aplicación de los SIG para realizar análisis de riesgos climáticos a través del uso de la cartografía. Así como la investigación de Gezan-Matus (2000), quien analiza la utilización de los SIG en protección civil, lo que es un aporte para el manejo de riesgos no solamente en la etapa de prevención, sino también en la de mitigación de desastres.

En este sentido, existe una propuesta de mapa de riesgos de bienes patrimoniales inmuebles expuestos a amenazas de origen natural, propuesto por Rueda (2014), en donde con la herramienta Model Builder de ArcGis se realizó el análisis y valoración del estado de conservación de los bienes patrimoniales inmuebles del Ecuador. Además de los estudios antes mencionados Chuvieco et al. (2005) definieron un nuevo término que hace uso de los SIG en el ámbito geográfico y lo denominaron Tecnologías de la Información Geográfica (TIG), para aplicarlo en ámbitos como la docencia, la investigación o el propio ejercicio profesional.

Criminología, donde se estudian conceptos como la modelización espacial y los análisis de patrones que interrelacionan fenómenos sociales, económicos, o ambientales con ocurrencias de delitos. Ejemplos de estudios en estas temáticas se pueden encontrar en la investigación de Vozmediano y San Juan (2006), referente al empleo de SIG en el estudio del miedo al delito, a través de los cuales se construyeron mapas de criminalidad en contextos urbanos.

$O$ en el estudio sobre criminología ambiental y SIG aplicado en una ciudad española, propuesto por Martínez (2016), en donde se realizó el análisis de los datos de los delitos y el espacio donde estos ocurren, facilitando el estudio del ambiente y sus características a fin de basar las actuaciones policiales en los análisis de dichos elementos, en este sentido se cuenta también con el estudio propuesto por Salafranca y Rodríguez (2014) en cuanto a SIG aplicados a la investigación policial, o el estudio de Alcívar y Calderón (2013), referente al análisis espacial y la distribución del delito en la ciudad de Guayaquil, en donde gracias a los métodos geoestadísticos se obtuvieron mapas de estimación y varianza a través de los cuales se conoció en que zonas se perpetran más delitos. Sin embargo este tipo de estudios no son completamente nuevos, ya que Stageland y Garrido (1999) hablaban sobre el análisis geográfico de la delincuencia urbana, en donde utilizaban medios informáticos para organizar información sobre el problema de la delincuencia.

Sociología, debido a que se estudia el comportamiento de los individuos y de los grupos que habitan en un determinado lugar. Así se pueden destacar el estudio de Barrera (2009) sobre los SIG participativos y la cartografía social, en donde se analizan aspectos sobre cartografía formal (euclidiana) y cartografía social (no necesariamente euclidiana) y su relación, con el objetivo de complementar estas dos formas de representación espacial de territorios.

En este mismo contexto se puede citar a Linares (2007) con su estudio sobre la aplicación de los SIG en la segregación socioespacial urbana, en donde el trabajo con bases de datos vinculadas a entidades geográficas georreferenciadas, permite realizar ajustes de la información desde puntos a líneas, y desde estos a polígonos, con lo cual se superan las limitaciones en relación a la forma en como aparecen almacenados los datos originalmente. Por otro lado, está el estudio de Chasco (2004), en referencia a la aplicación del 
geomarketing y la distribución comercial, en donde se realiza un análisis geográfico de la realidad económico-social a través de los SIG, para encontrar aquellas localizaciones de mayor potencialidad para el establecimiento de un negocio.

Se cuenta también con estudios respecto al uso de los SIG para la optimización de la administración pública (Gallego, Ramos \& Arámbula 2003), a fin de incluir información espacial y temática que permita llevar a cabo consultas de diversos tipos, así como ejecutar modelos cartográficos dinámicos.

Además de lo mencionado están estudios tan específicos como el planteado por Jiménez, Romeu, Pámies y Guasch (2008) que hablan sobre cómo gestionar de manera efectiva el ruido a través de la representación de los niveles sonoros utilizando dichos sistemas de información, o el de Becerra, Paichard, Sturma y Maurice (2013), quienes hablan de las percepciones sociales del riesgo sanitario por la contaminación petrolera en el Ecuador, y de cómo las circunstancias geográficas podrían limitar las posibilidades de actuación.

En este sentido, la aplicación de los SIG en las ciencias sociales, abarca diversos campos de estudio desde la arqueología, hasta la sociología, pasando por temas tan concretos como la economía y la criminología, lo que indica que las ciencias computacionales con las sociales, se complementan de manera perfecta para aportar a la ciencia y al conocimiento de los pueblos. Es indudable que el uso de estos sistemas de información son una tendencia en alza (Crespo 2013) debido a las facilidades que aporta en diversos campos de estudio como quedó demostrado en la revisión bibliográfica mencionada.

Así, se puede notar que en cada una de las ciencias de carácter social consideradas para esta investigación, existen estudios y publicaciones de gran interés en el contexto regional (Sudamérica), así como en el iberoamericano y porque no decirlo en el global.

A criterio de los autores, el descubrimiento y avance de muchas investigaciones en arqueología ha sido gracias a la utilización de los SIG por parte de los profesionales que realizan este tipo de estudios, esto a su vez ha permitido la diversificación de los métodos y técnicas utilizadas a la hora de realizar exploraciones arqueológicas, con lo cual el análisis de un territorio determinado se podría hacer en menos tiempo y con menor utilización de recursos.

De la misma manera, cuando nos referimos al aspecto físico y a las expresiones sociales y/o culturales de una población determinada, se constata la utilización de cartografías para determinar elementos antropológicos y su interrelación con el hábitat de los pueblos considerando incluso su flora y fauna como recursos que pueden ser vigilados $y$ administrados de mejor manera a través de la utilización de los SIG, lo cual es de suprema importancia debido a que hoy por hoy el aspecto cultural ha sido retomado y está siendo ampliamente utilizado para lograr el tan anhelado Sumak Kawsay, expresión kichwa, que significa Buen Vivir (Cortez 2017).

Así la realización de la llamada cartografía etnográfica, permitiría por ejemplo detectar de manera eficaz las manifestaciones culturales, sociales, económicas e incluso étnicas de una determinada población (Athabe 2006). Además, es importante mencionar la planificación territorial a fin de relacionar factores tales como el cuidado del medio ambiente, la preservación de la biodiversidad y el patrimonio cultural, además de potenciar el desarrollo sustentable de los habitantes de un determinado lugar.

En este punto, podemos mencionar también, que al estar utilizando una herramienta tecnológica, es indudable el aporte de los profesionales de la ingeniería en diversos ámbitos como el desarrollo de sistemas informáticos, las telecomunicaciones, arquitectura, ingeniería civil, agroindustrial, ambiental y demás. 
Hablando de temas como la economía o la geografía humana, los estudios encontrados sirven para la toma decisiones en cuanto a la gestión de un determinado territorio o de un grupo poblacional en particular, o en cómo la población podría ser distribuida en un territorio de tal manera de aprovechar más eficientemente los recursos naturales del sector, por ejemplo, para saber qué tan sustentable puede o no ser una economía de acuerdo con el medio ambiente existente.

En este sentido, los estudios relacionados con las áreas mencionadas, tiene como objetivo la preservación del hábitat a fin de prevenir cambios climáticos extremos, como el que lastimosamente ya estamos viviendo y que está relacionado con el calentamiento global debido al aumento de los gases de efecto invernadero como el dióxido de carbono, lo que produce entre otras cosas el desgaste de la capa de ozono (Caballero, Lozano \& Ortega 2007).

Pero además puede abarcar temas tan importantes como la seguridad alimentaria, tal como se refleja en un estudio de las Naciones Unidas para el Ecuador, o en la utilización de los SIG para prevenir y mitigar desastres, a efectos de proteger a la población.

Para el caso específico de ciencias como la criminología, el uso de SIG permite detectar patrones de conducta sobre una determinada región, con lo cual a criterio de los autores de este artículo, la efectividad de la investigación policial podría incrementarse. Pero además de estudiar el comportamiento social, los SIG son utilizados para conocer las ubicaciones precisas en donde se realizan más actos delictivos, lo que indudablemente podría servir para prevenir situaciones de riesgo tal como se detalla en Vázquez y Soto (2013), a través de la elaboración de mapas y la utilización de métodos y técnicas relacionadas con la geoestadística.

Por su parte en sociología, pensamos que el estudio del comportamiento de los seres humanos, apoyándose en los SIG ha revolucionado la concepción y la utilización misma de estas ciencias, por ejemplo, al acuñar términos como el de sistemas de información geográfica participativos y cartografía social, los cuales según algunos autores como Sastre, Dorado y Ríos (2010), fomentan el desarrollo sostenible, al trabajar con datos geo referenciados.

Se puede mencionar además la gestión del ruido en áreas urbanas, el estudio de la segregación socioespacial, la optimización de la administración pública, la aplicación del geomarketing, o como en el caso de Ecuador, de las percepciones sociales de la contaminación. Todos estos son buenos ejemplos de cómo la utilización de los SIG coadyuva de manera óptima a mejorar la calidad de vida de los seres humanos.

\section{CONCLUSIONES}

El aporte al conocimiento universitario planteado como objetivo para el desarrollo del presente artículo de revisión se ha hecho efectivo, debido a que se recopilaron, clasificaron, analizaron y sintetizaron trabajos relevantes en el área de las ciencias sociales, los cuales estuvieron soportados a través de la utilización de los SIG.

Como se puede colegir, por lo expuesto en el acápite de resultados y discusión de este artículo, la utilización de los SIG en el área de ciencias sociales, ha tenido un crecimiento importante, debido a la gran diversificación de estudios en esta área, los cuales utilizan la información geográfica como apoyo para el desarrollo de sus ciencias.

La aplicación de los SIG en las ciencias sociales, no solamente es útil en el ámbito académico, sino que también diferentes sectores como el social, productivo, de negocios, e incluso el comercial han obtenido provecho de la utilización de esta importante herramienta de tecnología, información y comunicación, tal como se demuestra en el desarrollo de este artículo.

La investigación basada en los SIG y aplicada a las ciencias sociales, tiene todavía mucho camino por recorrer, así los beneficios que 
pueden obtenerse de la utilización de dichos sistemas de información es infinita, lo que permitirá que la interrelación entre las ciencias de la ingeniería, las sociales y las de la educación en general se haga más fuerte.

\section{REFERENCIAS BIBLIOGRÁFICAS}

Aguirre, A. (2013). Sistema de infor- mación geográfica para la gestión de la bioseguridad en la provincia Holguín. Ciencia en su pc, 4, 103110. Recuperado de http://www. redalyc.org/pdf/1813/Resumenes/ Abstract_181332462008_2.pdf

Alcívar, C. \& Calderón, J. (2013). La delincuencia en la ciudad de Guayaquil, un análisis espacial de su distribución por delito. Revista Caribeña de Ciencias Sociales. Recuperado de http://xn--caribea-9za.eumed.net/ delincuencia-guayaquil/

Alonso, F. (2004). SIG aplicados al análisis y cartografía de riesgos climáticos. Recuperado de http://www.um.es/ geograf/sigmur/cursos/SIG_clima. pdf

Alonso, F. (2006). Sistemas de Información Geográfica. Recuperado de http:// www.um.es/geograf/sigmur/temariohtml/index.html

Athabe, G. (2006). Hacia una antropología del presente. Cuadernos de Antropología Social, 23, 13-34. Recuperado de https://dialnet.unirioja.es/descarga/articulo/5281938.pdf

Barrera, S. (2009). Reflexiones sobre Sistemas de Información Geográfica Participativos (sigp) y cartografía social. Cuadernos de Geografía, 18,
9-23. doi: 10.15446/rcdg.n18.12798. Recuperado de https://revistas. unal.edu.co/index.php/rcg/article/ view/12798/38453

Becerra, S., Paichard, E., Sturma, A. \& Maurice, L (2013). Vivir con la contaminación petrolera en el Ecuador: Percepciones sociales del riesgo sanitario y capacidad de respuesta. Revista Líder, 23, 102-120.

Burel, C., Rubió, J. \& Sitjár, J. (2012). Los SIG como instrumento para el análisis de las migraciones: el ejemplo del éxodo catalán de 1936. Diacronie, 10(2), 1-17. Recuperado de https:// journals.openedition.org/diacronie/2831

Caballero, M., Lozano, S. \& Ortega, B. (2007). Efecto invernadero, calentamiento global y cambio climático: Una perspectiva desde las ciencias de la tierra. Revista Digital Universitaria, 8(10), 1-11. Recuperado de http://132.248.9.34/hevila/Revistadigitaluniversitaria/2007/vol8/ no10/5.pdf

Carrera, G. (2007). La cartografía etnográfica como herramienta técnica y metodológica en investigaciones antropológicas. El caso de Constantina. Cuadernos de los Amigos de los Museos de Osuna, 9, 72-81. Recuperado de https://dialnet.unirioja.es/servlet/ articulo? codigo $=2885431$

Cerda, J. \& Valdivia, G. (2007). John Snow, la epidemia de cólera y el nacimiento de la epidemiología moderna. Revista chilena de in-fectología, 24(4), 331334. Recu- perado de https://scielo.conicyt. cl/scielo.php?script=sci_arttext\&pid=S0716-10182007000400014

Chasco, C. (2004). El Geomarketing y la distribución comercial. Investigación y Marketing, 79, 6-13. Recupera- 
do de https://www.researchgate.net/ publication/23749590_El_Geomarketing_y_la_distribucion_comercial

Chuvieco, E., Bosque, J., Pons, X., Conesa, C., Santos, J., Gutiérrez, J. \& Prados, M. (2005). ¿Son la tecnologías de la información geográfica (TIG) parte del núcleo de la geografía?, Boletín de la A.G.E, 40, 35-55. Recuperado de https://dialnet.unirioja.es/servlet/ articulo?codigo $=1308488$

Crespo, A. (2013). La Historia geográficamente integrada y los Sistemas de Información Geográfica (SIG): concepto y retos metodológicos. Tiempos Modernos, 7(26), 1-33. Recuperado de http://www.tiemposmodernos.org/tm3/index.php/tm/article/ view/331/373

Cortez, D. (2017). La construcción social del "Buen Vivir" (Sumak Kawsay) en Ecuador. Recuperado de: http:// www.uasb.edu.ec/UserFiles/369/ File/PDF/CentrodeReferencia/Temasdeanalisis2/buenvivirysumakkawsay/articulos/Cortez.pdf

Del Bosque Gónzález, I., Fernández Freire, C., Martín-Forero Morente, L. \& Pérez Asensio, E. (2012). Los sistemas de información geográfica y la Investigación en Ciencias Humanas y Sociales. Madrid, España: Eds. Confederación Española de Centros de Estudios Locales (CSIC).

Enríquez, K., Alfaro, E., Juncosa, J., Delgado, F., Rivadeneira, M., Chala, J. \& Viteri, A. (2011). La arqueología y la antropología en Ecuador: Escenarios, retos perspectivas. Recuperado de https://dspace.ups.edu.ec/handle/123456789/5647

ESRI (2017). Overview. Recuperado de http://www.esri.com/about-esri\#what-we-do

Fagandini, F. \& Villanueva, A. (2012). Los
SIG y la economía ambiental herramientas para la toma de decisiones territoriales. Caso de estudio El fundo Carmen, comuna de Quilipué, Chile. Revista geografía de Valparaiso, 45, 23-32. Recuperado de https://www.researchgate.net/publication/281108647_Los_SIG_y_la_ economia_ambiental_herramientas_para_la_toma_de_decisiones_ territoriales_Caso_de_estudio_Fundo_el_Carmen_Comuna_de_Quilpue_Chile

Figuerero, M. (2018). Tecnología e ideas usadas para el análisis espacial de paisajes arqueológicos en la Argentina. Arqueología, 24(3), 185-192. Recuperado de http://revistascientificas. filo.uba.ar/index.php/Arqueologia/ article/view/5413/4859

Figuerero, M. \& Izeta, A. (2013). El uso de Sistemas de Información Geográfica (SIG) en arqueología sudamericana . Recuperado de http://sedici.unlp.edu. ar/bitstream/handle/10915/27415/Introducci\%C3\%B3n.pdf?sequence=1

Gallego, M., Ramos, L. \& Arambula, S. (2003). Sistemas de Información Geográfica para la Optimización de la Administración Pública. Revista de Ingeniería, 47(17), 39-48. Recuperado de https://ojsrevistaing.uniandes.edu.co/ojs/index.php/revista/ article/view/498

García, J. (1976). Antropología del territorio. Madrid, España: Taller de Ediciones Josefina Betancourt.

Gezan-Matus, T. (2000). Sistemas de información geográfica aplica- dos en protección civil. Revista de Geografía Norte Grande, 27, 103109. Recuperado de https:// repositorio.uc.cl/bitstream/handle/11534/10437/000313772.pdf?sequence $=1 \&$ isAllowed $=\mathrm{y}$ 
Goerlich, F. \& Cantarino, I. (2013). Geodemografía: coberturas del suelo, sistemas de información geográfi- ca y distribución de la población. Investigaciones regionales, 25, 165191. Recuperado de https:// old.aecr.org/images//ImatgesArticles/2013/5/8_Goerlich.pdf?_ $\mathrm{ga}=2.217193237 .2011544584$. 1555429673 - 324614149 . 1555429673

Haro-Martínez, A. \& Taddei-Bringas, I. (2014). Sustentabilidad y economía: la controversia de la valoración ambiental. Economía, sociedad y territorio, 14(46), 1-15. Recuperado de http://www.scielo.org.mx/pdf/est/ v14n46/v14n46a7.pdf

Hernández, D. \& Witter, R. (2011). Entre la ingeniería y la antropología: haciaun sistema de indicadores integrado sobre transporte público y movilidad. Revista Transporte y Territorio, 4, 29-46. Recuperado de http://revistascientificas.filo.uba.ar/index.php/rtt/ article/view/255/233

Ibarra, G. (2013). Aplicaciones del siste- ma de información geo referencia- do en el Ecuador. Revista Científica YACHANA, 2(2), 279-282. Recuperado de http://revistas.ulvr.edu.ec/index.php/yachana/article/view/66/60

Jiménez, S., Romeu, J., Pámies, T. \& Guasch, S. (2008). Sistemas de Información Geográfica en la gestión integral del ruido. Acústica, 1-11. Recuperado de http://www.sea-acustica.es/fileadmin/Coimbra08/id037.pdf

Linares, S. (2007). Aplicación de sistemas de información geográfica al estudio de la segregación socioespacial urbana: el caso de la ciudad de Tandil. (Tesis de maestría, Universidad Nacional del Centro de la Provincia de Buenos Aires, Argentina). Recuperado de https://www.researchgate.
net/profile/Santiago_Linares/publication/299579770_Aplicacion_de_ Sistemas_de_Informacion_Geografica_al_estudio_de_la_segregacion_ socioespacial_urbana_el_caso_de_ la_ciudad_de_Tandil/links/570047e308aea6b77469b797.pdf.

Maldonado, G. (2012). El uso de los SIG para la enseñanza de Ciencias Sociales en la Educación Secundar- ia Informe. Recuperado de http:// repositorio.ual.es:8080/bitstream/ handle/10835/2018/TRAB A JO_7035_1064.pdf?sequence $=1 \&$ isAllowed $=\mathrm{y}$

Malo, A. (2009). El territorio, el desarrollo sustentable y los sistemas de información geográfica. Geomática, 49, 81-96.

Martínez Roig, A. (2016). Criminología Ambiental y SIG, una aplicación práctica en Castellón de la Plana. (Trabajo de fin de grado, Universitat Jaume I, Castellón, España). Recuperado de http://repositori.uji.es/xmlui/bitstream/handle/10234/161313/TFG_ Marti\%CC\%81nez\%20Roig_Angel. pdf? sequence $=1 \&$ isAllowed $=\mathrm{y}$

Pastor, S., Murrieta, P. \& García, L. (2013). Los sig en la arqueología de habla hispana. Temas, técnicas y perspectivas. Comechingonia, 17(2), 9-29. Recuperado de https://ri.conicet.gov. ar/bitstream/handle/11336/25299/ CONICET_Digital_Nro.4a8b6d059252-4a12-9fcf-6a8fd7b67b29_A. pdf? sequence $=2 \&$ isAllowed $=y$

Rivas, P. \& Perera, M. (2008). La etnocartografía y el Sistema de Información Geográfico Ecológico Cultural del pueblo Pemón (SIGEC-Pemón). Alcances y posibilidades. Antropológica, 109, 27-67. Recuperado de https://biblat.unam.mx/es/revista/ antropologica-caracas/articulo/la-etnocartografia-y-el-sistema-de-infor- 
macion-geografico-ecologico-cultural-del-pueblo-pemon-sigec-pemon-alcances-y-posibilidades

Rodríguez-Pérez, S. (2015). SIG y Gestión de Bases de Datos: una aplicación en el estudio arqueológico de la arquitectura tradicional del occidente asturiano. Sémata, Ciencias Sociales y Humanidades, 27, 239-264. Recuperado de http://www.usc.es/ revistas/index.php/semata/article/ view/2953/3283

Rosero, C., Vásquez, P. \& Cordero, V. (2010). Análisis situacional de la soberanía alimentaria en el contexto de la adaptación al cambio climáti- co en el Ecuador. Informe Técnico Definitivo. Recuperado de https:// www.undpcc.org/docs/National\%20 issues \%20papers/Agriculture $\% 20$ (adaptation)/04_Ecuador\%20NIP_ food $\% 20$ security $\% 20$ adaptation.pdf

Rueda, E. (2014). Propuesta de Mapa de Riesgos de Bienes Patrimoniales Inmuebles expuestos a Amenazas de Origen natural. Enfoque UTE, 5(1), 30-48. doi:10.29019/enfoqueute. v5n1.34. Recuperado de http://ingenieria.ute.edu.ec/enfoqueute/index. $\mathrm{php} / \mathrm{revista/article/view/34}$

Salafranca, D. \& Rodríguez, M. (Junio de 2014). Sistemas de información geográfica aplicados a la investigación policial. En A. Ramón (Presidencia), XVI Congreso Nacional de Tecnologías de la Información Geográfica, Alicante, España.

Saldarriaga, C. (2007). SIG en el análisis económico del manejo y conservación del medio ambiente y los recursos naturales. Ensayos de economía, 31, 125-136. Recuperado de https://revistas.unal.edu.co/index.php/ede/article/viewFile/25012/25549

Santovenia, J., Tarragó, C. \& Cañedo, R.
(2009). Sistemas de información geográfica para la gestión de la información. ACIMED, 20 (5), 7275. Recuperado de http://scielo.sld. cu/scielo.php? script=sci_arttext\&pid=S1024-94352009001100007

Sartori, J., Fernando, S. \& Colasurdo, M. (2017). El espacio y los recursos: el análisis arqueofaunístico mediante SIG en los distintos ambientes de la provincia de Santa Fe. Antípoda. Revista de Antropología y Arqueología, 28, 99-125. Recuperado de https://www.redalyc.org/articulo. oa? $\mathrm{id}=81451534006$

Sastre, S., Dorado, G. \& Ríos, I. (Junio-Julio de 2010). Los sistemas de información geográfica participativos como herramientas para el desarrollo rural sostenible: Análisis conceptual y revisión de experiencias. En

A. Cazorla (Presidente), 1st Latin American Conference on Project Engeneering, llevada a cabo en el XIV International Congress on Project Engineering, Madrid, España.

Stageland, P. \& Garrido, M. (1999). El análisis geográfico de la delincuencia urbana. Boletín Criminológico, 41,1-4. Recuperado de http://www.boletincriminologico.uma.es/boletines/41. pdf

Ther, F. (2012). Antropología del terri- torio. Revista Latinoamericana Polis, 13 (32), 493-510. Recuperado de https://scielo.conicyt. cl/scielo.php?script $=$ sci_arttext\&pid=S0718-65682012000200023

Vásquez, C. \& Soto, C. (2013). El análisis geográfico del delito y los mapas de la delincuencia. Revista de derecho penal y criminología, 3(9), 419-448.

Vozmediano, L. \& San Juan, C. (2006). Empleo de Sistemas de Información Geográfica en el estudio del Miedo 
al Delito. Revista Española de Investigación Criminológica, 4, 1-11. Recuperado de https://dialnet.unirioja. es/servlet/articulo?codigo $=2083345$ 